\title{
Development of a low-cost micro-CMM for 3D micro/nano measurements
}

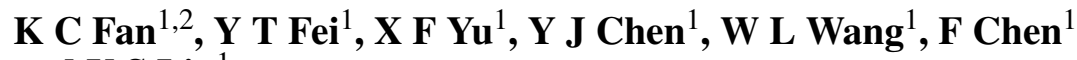 \\ and Y S Liu ${ }^{1}$ \\ ${ }^{1}$ Department of Precision Instruments, Hefei University of Technology, Hefei 230009, \\ People's Republic of China \\ ${ }^{2}$ Department of Mechanical Engineering, National Taiwan University, 1, Sec. 4, \\ Roosevelt Road, Taipei 10627, Taiwan \\ E-mail: fan@ntu.edu.tw
}

Received 8 June 2005, in final form 22 September 2005

Published 31 January 2006

Online at stacks.iop.org/MST/17/524

\begin{abstract}
A high-precision and low-cost micro-CMM (coordinate measuring machine) is under development. The expected measuring range is $25 \times 25 \times 10 \mathrm{~mm}^{3}$ and the resolution is $1 \mathrm{~nm}$. In order to enhance the structural accuracy, some new ideas are integrated into the design, such as the arch-shape bridge for better stiffness and thermal accuracy, and the co-planar stage for less Abbe error. The linear diffraction grating interferometer and subdivision technique is proposed for position sensing to nanometre resolution. The focusing probe on the laser interferometer feedback spindle is structured in the $Z$-axis to guarantee the nanometre stability. In this report, the detailed design principles of the developed micro-CMM are described. The performance evaluation of each module of the prototype micro-CMM is presented. The positioning resolution of each axis to $1 \mathrm{~nm}$ can be achieved by combining the coarse and fine motion control on a piezo-ceramic linear motor. The $Z$-axis measurement can be controlled to within $15 \mathrm{~nm}$ repeatability. Parts of the positioning repeatability of the co-planar stage have been achieved to $30 \mathrm{~nm}$. Some problems due to current techniques will be addressed.
\end{abstract}

Keywords: Micro-CMM, nanometre accuracy, arch bridge, co-planar stage, focusing probe

(Some figures in this article are in colour only in the electronic version)

\section{Introduction}

The technology of micro/nano 3D profile measurement has received a great deal of attention during the past decade [1]. Many fine components recently fabricated by micro system processes, such as MEMS, LIGA or micro machining, are in overall dimensions within meso scale and required accuracy from microns to tens of nanometres. Conventional coordinate measuring machines (CMM) are no longer capable of 3D measurements of these fine parts. Some advanced probes, such as SPM, are already commercially available but are limited to only $1 \mathrm{D}$ sensing to nanometre resolution. Even equipped with PZT stages their measurement ranges are limited up to $100 \mu \mathrm{m}$. It was noted by Takamasu [2] and Ni [3] that an overall consideration in the 3D measurement system and its integration should not be overlooked. Small-sized CMMs, such as the nano-CMM by Takamatsu [2], small CMM by NPL [4], nanopositioning CMM by Hausottee and Jäger [5], micro-CMM by Fan [6] and Liang [7], etc, have become a new topic of research. There are also some micro-scaled touch probe systems, such as the mechanical ball by Takamasu [8], MEMS by Eindhoven University [9], optical fibre by PTB [10], etc. Small CMM requires higher accuracy and resolution. Scaling down the conventional CMM design principle is not a feasible way as many existing mechanical components are all in micrometre accuracy. This paper presents an innovative micro-CMM design with the consideration of some completely new system modules, including the arch-shape bridge for 


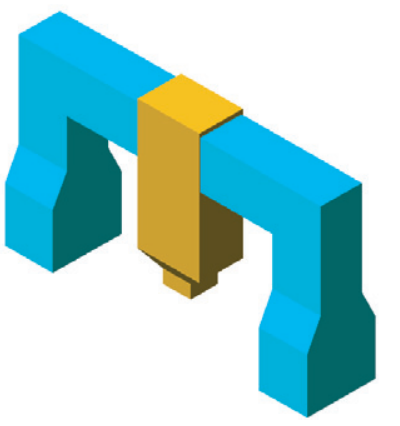

(a)

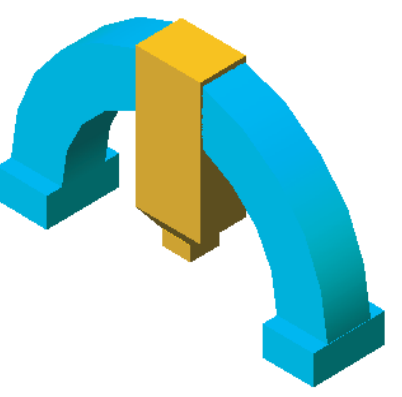

(b)

Figure 1. (a) The rectangular bridge and $(b)$ the arch bridge.

better stiffness and thermal accuracy, the co-planar stage for less Abbe error, the diffraction gratings with interferometric fringes and subdivision technique for nanometre resolution, and the focusing probe on the laser interferometer feedback spindle to guarantee nanometre stability. This micro-CMM is designed for the measurement of meso-to-micro scale parts. It is aimed at achieving $1 \mathrm{~nm}$ resolution and $30 \mathrm{~nm}$ repeatability within a measuring range of $25 \times 25 \times 10 \mathrm{~mm}^{3}$. So far, the $Z$-axis measurement can be controlled to within $15 \mathrm{~nm}$ repeatability. Parts of the objectives have been achieved. Some problems due to current techniques will be addressed.

\section{System configuration of the micro-CMM structure}

\subsection{The arch-bridge structure}

A rectangular type of bridge is always employed in the precision CMM structure for mounting the $Z$-axis probe, as shown in figure 1(a). Although its static deflection does not influence the measuring accuracy, the generated driving force and temperature rise from the motion actuator will, however, induce dynamic and thermal deformations of the bridge up to submicron level. In order to meet the high-precision requirement in nanometre measurement, the conventional rectangular-bridge shape has to be redesigned.

The deformation at the centre of the bridge is very critical because of the concentrated load from the spindle and the generated driving force, which will react to the bridge. The maximum deflection at the centre of the rectangular bridge due to the spindle load $P$ can be described as [15]

$$
\delta_{y \max }=0.55 \frac{P R^{3}}{E I}
$$

where $R$ is the half span of the bridge, $E$ is Young's modulus of the bridge material and $I$ is the moment of inertia of the cross section. During the spindle up and down motion, the generated driving force of the actuator will impose a dynamic force $(p)$ on the bridge. The overall load on the bridge centre will be $(P+\boldsymbol{p}) . \boldsymbol{p}$ can be expressed by $p \sin (2 \pi f t)$, where $p$ is the amplitude of the dynamic force and $f$ is the frequency.

This research proposes a fixed arch-bridge structure, as shown in figure $1(b)$. Under the same dimension and the same spindle load, the maximum deflection at the centre of the arch

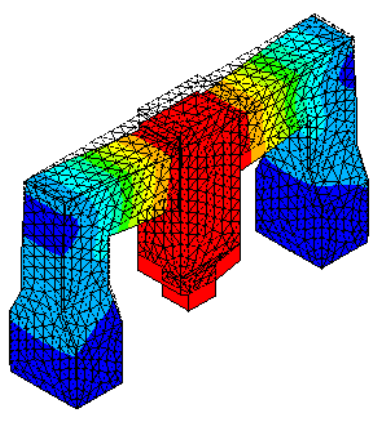

(a)

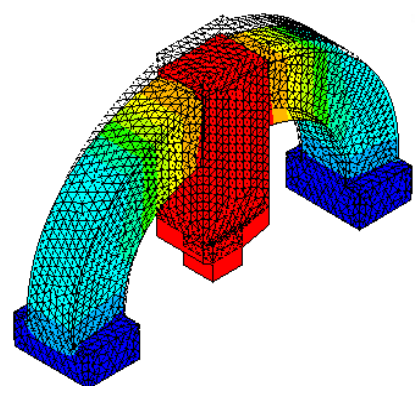

(b)
Figure 2. Deformed bridges by FEM analysis: (a) rectangular, (b) arch.

Table 1. Comparison between rectangular bridge and arch bridge $(\mu \mathrm{m})$.

\begin{tabular}{llll}
\hline & \multirow{2}{*}{$\begin{array}{l}\text { Analytical solution } \\
\text { weflection }\end{array}$} & \multicolumn{2}{c}{ FEA } \\
\cline { 3 - 4 } & & Spindle load & Self-weight \\
\hline Rectangular & 0.398 & 0.362 & 0.156 \\
Arch bridge & 0.197 & 0.174 & 0.102 \\
\hline
\end{tabular}

bridge can be reduced to ( $R$ is the radius of the semi-circle bridge):

$$
\delta_{y \max }=0.24 \frac{P R^{3}}{E I} .
$$

The physical dimensions of the developed micro-CMM bridge are as follows: the outer radius is $220 \mathrm{~mm}$ and the inner is $150 \mathrm{~mm}$; the width is $60 \mathrm{~mm}$ and the dimensions of the supporting pad are $70 \mathrm{~mm} \times 100 \mathrm{~mm} \times 40 \mathrm{~mm}$. It is made of granite material. The total weight is about $40 \mathrm{~kg}$. The spindle adds an additional weight of about $3 \mathrm{~kg}$. Figure 2 shows the deformed shapes of two bridges by the finite element method. Table 1 lists the comparison between the analytical and FEM methods of the two bridges.

From table 1 we can clearly see that the arch bridge has higher stiffness than the conventional rectangular type to almost twice the amount. Although the static stiffness does not influence the accuracy of static measurement, however, the imposed dynamic force will certainly impose dynamic deflection on the bridge during the spindle motion for the touch-triggered or scanning measurement mode. The final dynamic force will still apply to the bridge at the spindle holding position. This research employs a piezo-ceramic ultrasonic actuator (Nanomotion Co., Model SP-4) to drive the spindle motion at the lower position of the spindle, being a single load condition. Its maximum excited force at maximum acceleration is $15 \mathrm{~N}$. The actual motion will be, however, controlled at a very low speed. Suppose the driving force is $1 \mathrm{~N}$; the corresponding dynamic Z-deflection at the spindle end will be $6 \mathrm{~nm}$ to the rectangular bridge, and $3 \mathrm{~nm}$ to the arch type, from ANSYS results.

With regard to the thermal deformation, the SP-4 generates heat due to its friction force. Experimental observation showed that for a $2 \mathrm{~h}$ low speed run of the SP4 at a normal ambient temperature of $23.5^{\circ} \mathrm{C}$ its surface temperature will increase by $1{ }^{\circ} \mathrm{C}$. This temperature rise will result in spindle expansion at the tip by an amount of $14.6 \mathrm{~nm}$ 


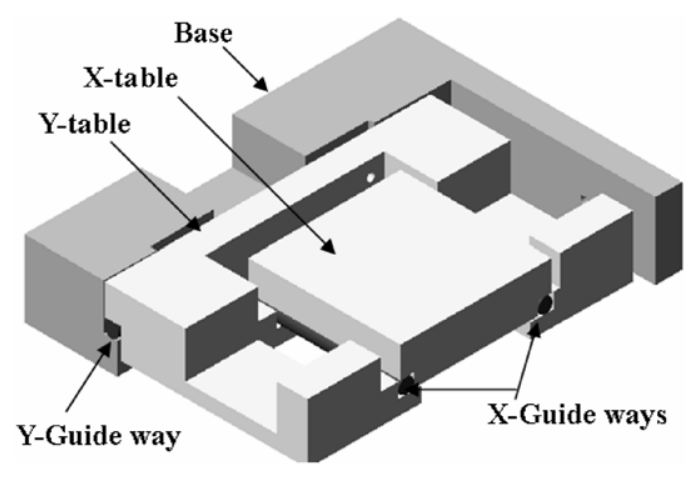

Figure 3. Proposed symmetrical co-planar $X Y$ stage.

for the rectangular-bridge and $9.1 \mathrm{~nm}$ for the arch bridge using the ANSYS analysis. Therefore, a well temperature controlled environment $\left(20 \pm 0.1^{\circ} \mathrm{C}\right)$ should be provided to reduce this thermal effect.

\subsection{The co-planar $X Y$ stage}

A conventional $X Y$ stage is normally stacked up by two linear stages composed of many components, such as ball screw, bearing, linear slide, etc. The Abbe error of the lower stage is high and the components are all made in micrometre accuracy range only. More rigorous considerations should be taken into account when the $X Y$ stage is used to micro/nano motion accuracy. An innovative co-planar stage is thus proposed in this study, as shown in its sectioning view in figure 3 . The top table is moved in the $X$-direction along the precision ground rods (or guideway) mounted onto the frame, and the frame is moved in the $Y$-direction along the precision ground rods of the base. The sliding surface of the moving part is mounted with a Teflon pad to reduce the friction. Four guiding rods (two for the upper axis and the other two for the lower axis) are located in the same plane, which means they share the same vertical height. This is the essence of the co-planar stage that the Abbe error in the vertical direction can be significantly reduced [20]. In addition, there are no transmission components and the geometry is symmetrical, which ensures less random error and better static deformation under the same working conditions. Each axis motion is actuated by a motor from one side and detected by a position feedback system from the opposite side, as shown in figure 5 .

The whole stage is made of Invar steel so that the thermal deformation due to the driving heat can be significantly reduced. In order to minimize the moving weight, the static deformation and the thermal deformation, a modified structure is redesigned. Figure 4 shows the new table shape of which the V-flat guideway feature allows the free end to eliminate the reaction force from the corresponding rod and allows free expansion of the table due to the driving heat. From the finite element analysis with ANSYS software it shows that at the table centre the static deformation is about $0.13 \mu \mathrm{m}$, and the thermal distortion is only $3.2 \mathrm{~nm}$ assuming a $5{ }^{\circ} \mathrm{C}$ temperature rise at the driver. Similar modification is also made to the moving frame. The complete design of this co-planar stage with minimum stress and weight condition is illustrated in figure 5, where the actuator and the position sensor will be described in the following sections.

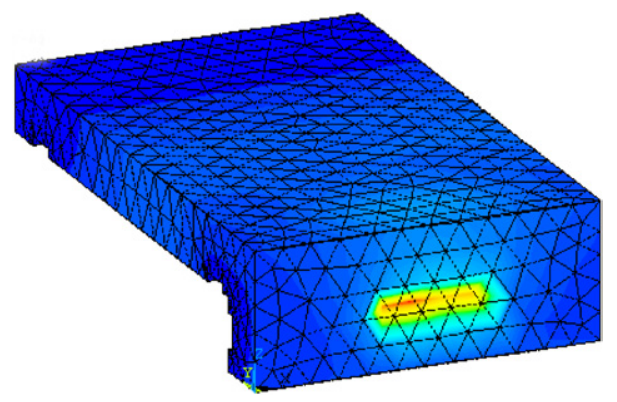

Figure 4. Modified guide way shape of the table.

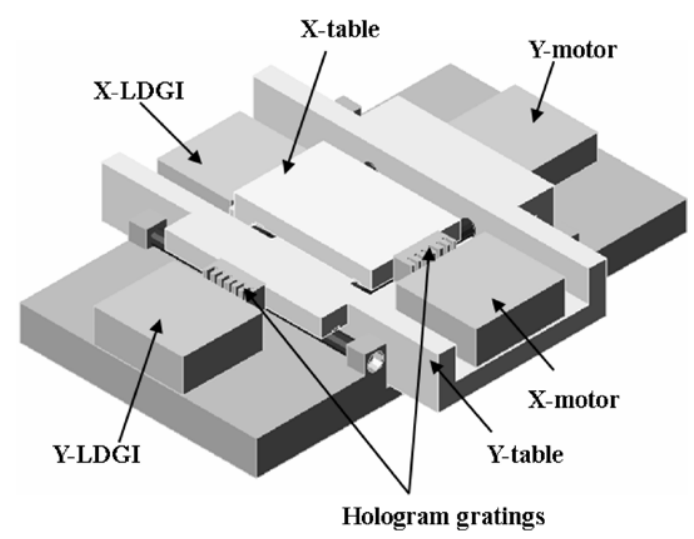

Figure 5. Final stage design of minimum stress and weight.

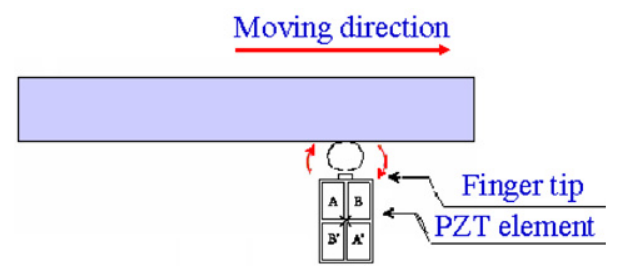

Figure 6. Motion principle of the ultrasonic motor.

\subsection{The driving system and feedback sensor}

In order to maintain high motion accuracy, the co-planar $X-Y$ stage and the $Z$-stage are all driven by ultrasonic motors (model SP-4 made by Nanomotion Co. [11]). The SP-4 system consists of the motor and a drive amplifier. These two components are combined to create the piezoelectric effect. This effect converts the electrical field to a mechanical motion. The four piezo-ceramic elements play an important role in the operation. When the excited voltage is applied across the element in a precise sequence, the front tip of the piezo elements generates an elliptical motion with the frequency of $39.6 \mathrm{kHz}$. This elliptical motion then drives the stage by a friction force to create a linear motion of the stage. The operational principle of this ultrasonic motor is shown in figure 6 where the tip is deliberately separated from the slide to show its elliptical motion form. This cyclic motion is called ac mode motion with a minimum step of $5 \mathrm{~nm}$. This ultrasonic motor also features a dc mode motion actuated by a dc voltage, which is proportional to an even finer motion within $5 \mathrm{~nm}$. Since the motor is tiny and easy to control it is suitable to small nanostages. 


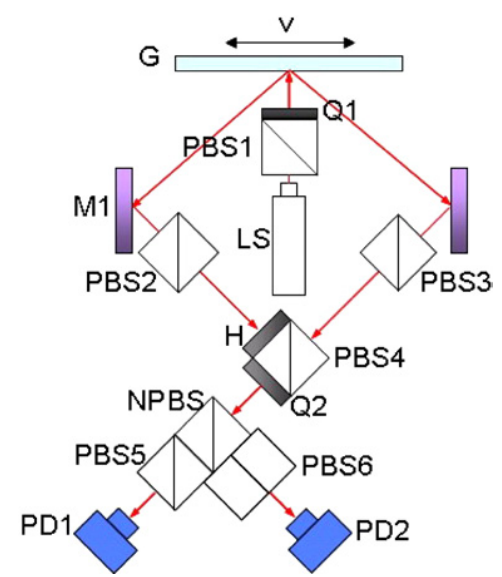

Figure 7. Principle of linear diffraction interferometer (LS: laser diode, L: lens, S: stage, G: grating, PBSi: ith polarizing beam splitter, Mi: ith mirror, NPBS: non-polarizing beam splitter, Q $i$ : $i$ th quarter wave plate $i, \mathrm{PD} i$ : $i$ th photo detector).

The position feedback of linear motion in each axis is detected by the principle of linear diffraction grating interferometer (LDGI) with a $1 \mathrm{~nm}$ resolution, as shown in figure 7 [12]. The laser diode emits a linearly P-polarized laser beam with $635 \mathrm{~nm}$ wavelength. The gratings will reflect with \pm 1 diffraction beams to mirrors 1 and 2, respectively. Passing through respective PBS (2 or 3 ) each beam will change to P-beam again. The left-arm beam changes to S-beam after it transmits through the half wave plate $(\mathrm{H})$. After the quarter wave plate Q2, the two diffractive beams will be retarded to the left-circularly polarized and right-circularly polarized beams, respectively. Again, passing through PBS5 and PBS6 the vectors of the electric field of the combined beams received by PD1 and PD2 will have a $90^{\circ}$ phase shift. The Doppler effects due to the motion of the grating will then shift the phase of each received beam with the wavelength proportional to the grating pitch. Meanwhile, the zero-order diffraction beam reflected from the gratings will be polarized to the S-linear beam so that it will not return to the laser diode to disturb its constant power.

The interferometer fringes always have three major errors: the dc shift difference, the electronic gain difference and the phase orthogonal error of two sinusoidal output signals [16]. Before going to the subdivision technique for finer resolution and accuracy, these errors have to be removed. In this research, the dc shift was compensated by the summation of each signal and its inverse signal to obtain the zero dc voltage; the gain error was removed by adjusting the respective resistance of each signal board and the orthogonal error of two signals was corrected by changing the outputs to their vector sum and difference. Figure 8 shows the Lissajous plots of before and after error compensation. The output signals have been modified very well for the further subdivision process.

With regard to the fringe subdivision technique, although there have been many methods proposed by various researches, such as curve fitting [16, 17], signal modulation [18] and CCD [19], this research developed a simple and quick method of triangulation form approximation by computing $|\sin \theta|-|\cos \theta|$ from the output signals, as shown in figure 9. The complete cycle counting and directional sensing can be done by a conventional up/down counter. Only the incomplete cycles at the beginning and the end $\left(X_{0}\right.$ and $\left.X_{1}\right)$ are to be interpolated. The calculation is as simple as linear interpolation. The approximated linearity is about $4 \%$.

\subsection{Spindle head design}

The spindle that carries the probe is moved along a short linear stage, which is driven by a PCLM and its motion is detected by a laser interferometer, as shown in figure 10. A counterweight

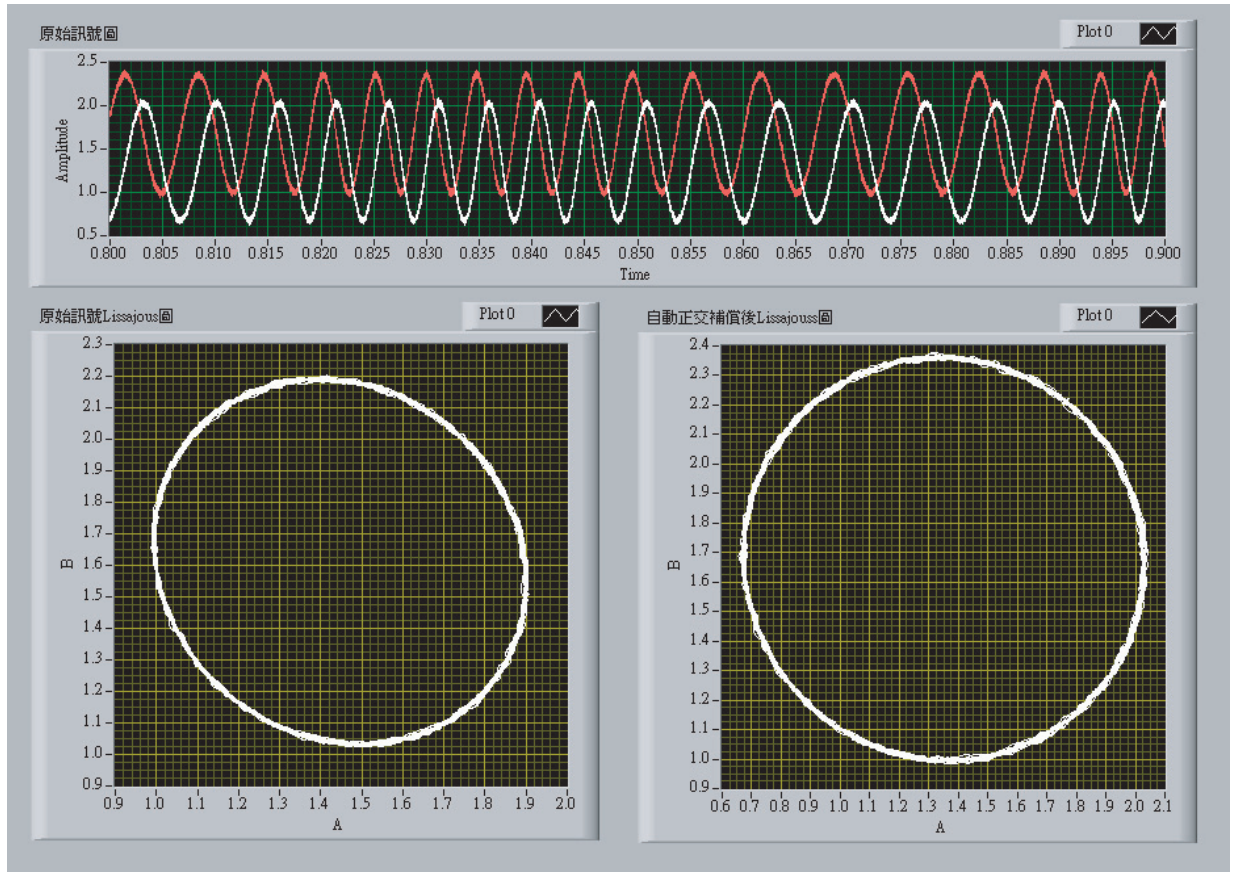

Figure 8. The Lissajous plots of before and after error compensation. 

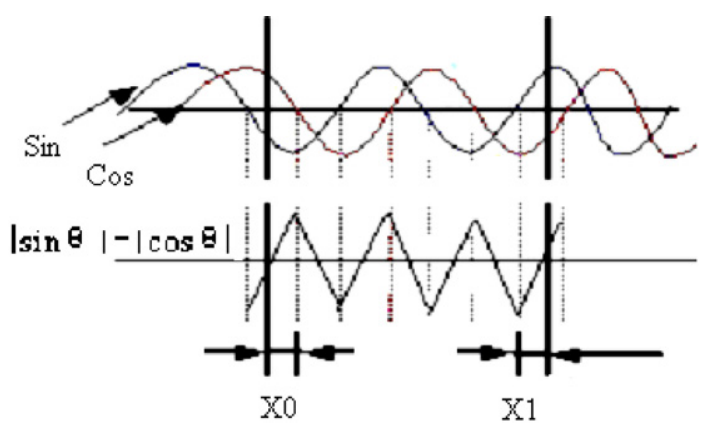

Figure 9. Signal subdivision method.

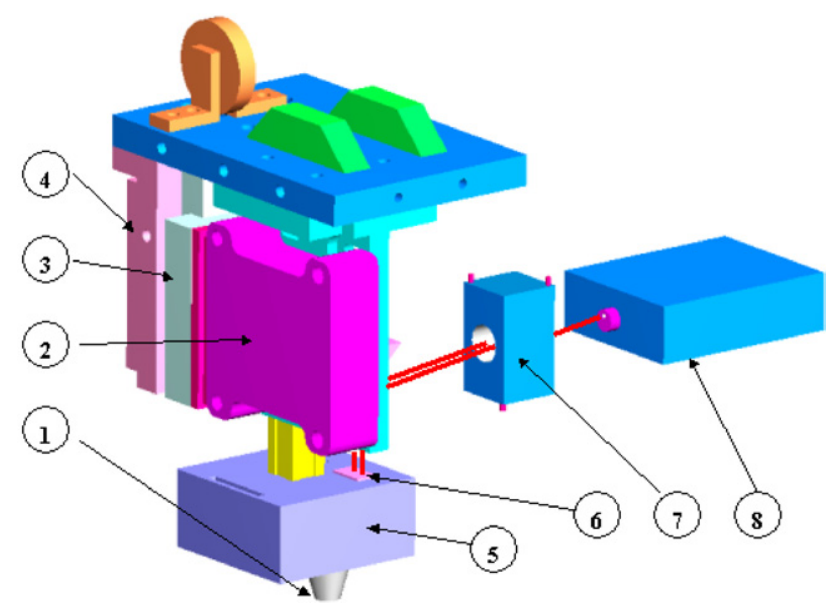

Figure 10. The spindle head design (1-probe tip, 2-PCLM, 3-linear stage, 4-counterweight, 5-probe head, 6-laser beam, 7-interferometer, 8-laser head).

is applied to balance the total mass centre during the spindle motion. The laser beam is in line with the probe to observe the law of Abbe's principle. During the motion the position is recorded by the laser interferometer and stopped by the probe at its focusing point on the object surface.

\section{Development of a focusing probe}

This research aims at the development of a low-cost optical probe with measurement capability in the nanometre range [13]. The pickup head of a commercial DVD player was adopted based on its principle of focus error. As shown in figure 11, a $635 \mathrm{~nm}$ wavelength light source generated from a laser diode is primarily polarized by a grating plate. After passing through a beam splitter and a quarter wave plate the light beam is focused by an objective lens onto the object surface with a spot size approximately $1 \mu \mathrm{m}$ in diameter. The reflected beam signal is imaged onto a four-quadrant photo detector through the quarter wave plate. The quadrant detector outputs are combined to give a focus error signal (FES). In this system, the focusing signal is detected by the astigmatic method. At the focal plane the spot is a pure circle. When the object moves up or down away from the focal plane, the spot takes an elliptical shape in different orientations. The corresponding FES provides an S-curve signal proportional to the distance, as shown in figure 12. The linear range of the S-curve will vary according to the object's surface

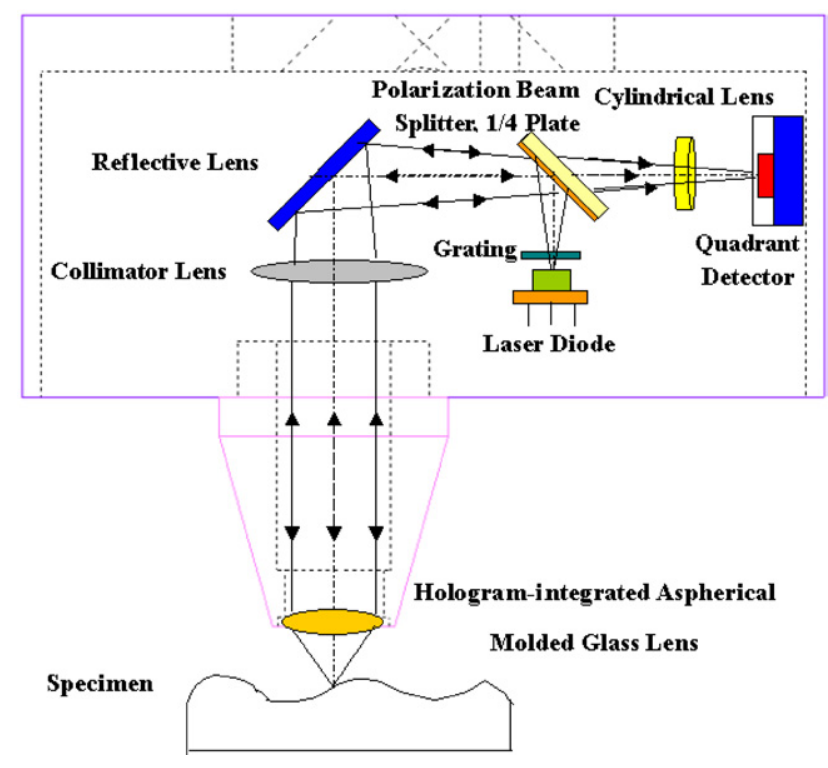

Figure 11. Principle of the focusing probe.

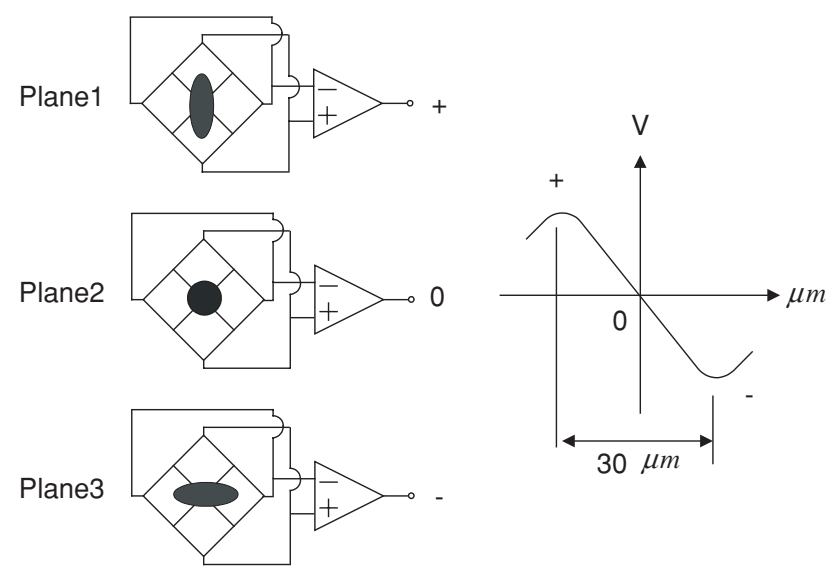

Figure 12. The variation of the spot shape with the S-curve.

reflectivity. The higher the reflective surface the larger the linear range.

\section{Experimental tests}

The developed micro-CMM has been fabricated in components and integrated into a prototype machine. Figure 13 shows the complete design and figure 14 is the prototype of the developed micro-CMM. Some performance tests have been carried out.

\subsection{Environmental effect}

Concerning the measurement in the nanometre scale the environmental effect is very critical to the measured data. For the reference length using a laser interferometer the temperature variation is the most important factor. This study was carried out in a temperature-controlled room. The current facility can, however, provide only up to $20 \pm 1{ }^{\circ} \mathrm{C}$ temperature control. Two types of laser interferometers were employed: one was HP5529A and the other (model PT-1002A) was made 
Table 2. Positioning accuracy of long travels (nm).

\begin{tabular}{lcccccc}
\hline $\begin{array}{l}\text { Position } \\
(\mathrm{mm})\end{array}$ & Error 1 & Error 2 & Error 3 & Error 4 & $\overline{\text { Error }}$ & $\sigma_{n-1}$ \\
\hline 2 & -49 & -29 & -54 & -46 & -44.5 & 10.8 \\
5 & 210 & 205 & 203 & 228 & 211.5 & 11.4 \\
10 & 708 & 678 & 697 & 708 & 697.7 & 14.2 \\
15 & 1955 & 1926 & 1970 & 1947 & 1950 & 18.3 \\
\hline
\end{tabular}

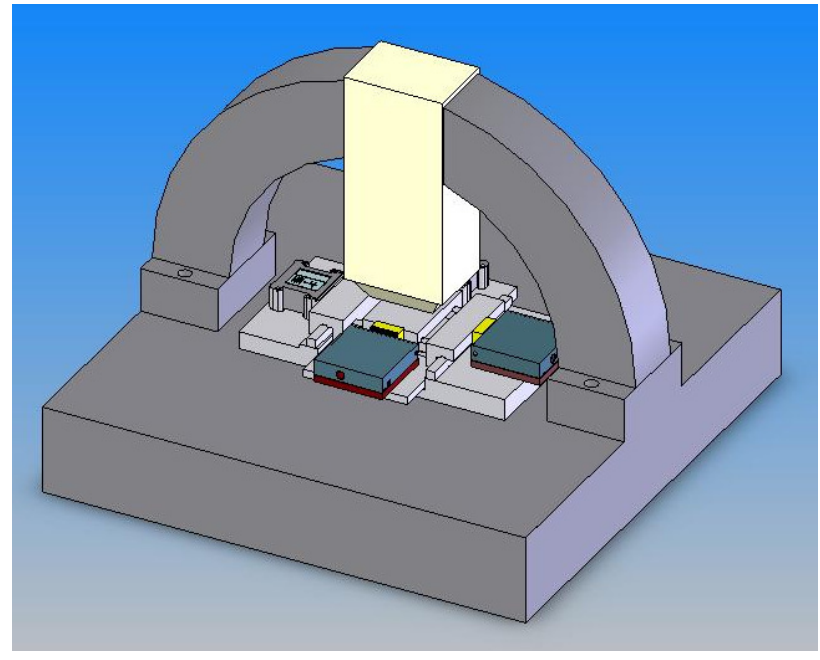

Figure 13. The structure of the innovative micro-CMM.

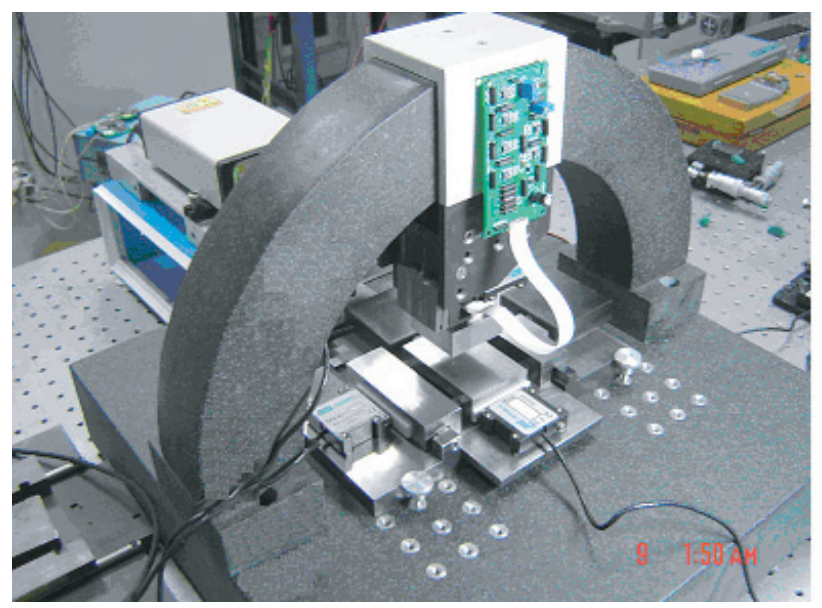

Figure 14. The prototype micro-CMM.

by Pretios Co. of China ${ }^{3}$. Both readings showed apparent drifts after warm-up and became stable after $3 \mathrm{~h}$. A stability test was then carried out to see the readings at every $10 \mathrm{~s}$ for $1800 \mathrm{~s}$. Figure 15 shows that HP5529A fluctuates between $60 \mathrm{~nm}$ and $-20 \mathrm{~nm}$, and PT-1002 varies between $18 \mathrm{~nm}$ and $-30 \mathrm{~nm}$. These results are not satisfactory yet. Hence, the environment control should be stricter.

\subsection{Positioning accuracy tests}

The motion of each axis, detected by the LDGI system, was calibrated using the HP5529A laser interferometer in a

\footnotetext{
3 Pretios Co., Beijing China, http://www.pretios.com, 2005.
}

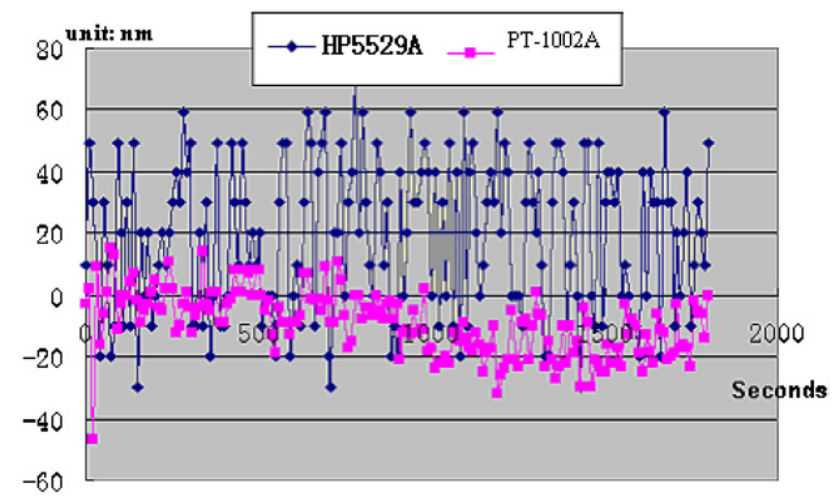

Figure 15. Stability tests of two laser interferometers.

temperature-controlled room $\left(20 \pm 1{ }^{\circ} \mathrm{C}\right)$. For the long travel test in the $Y$-direction, the motion was started by the ac mode and stopped with the dc mode of the ultrasonic motor. Results of four runs for each position from $2 \mathrm{~mm}$ to $15 \mathrm{~mm}$ are listed in table 2. Accumulated errors are clearly seen due to the geometrical errors of the axis motion. These systematic errors shall be compensated in the future. Standard deviations are in the range from $11 \mathrm{~nm}$ to $18 \mathrm{~nm}$. The repeatability, or $95 \%$ uncertainty, is slightly beyond $30 \mathrm{~nm}$ after $10 \mathrm{~mm}$ travel. Some possible error sources were found as follows.

1. The straightness polish of the guideway contact was not satisfactory. The induced variable friction forces yielded unsteady motion, which caused the noisy signals of the LDGI output.

2. The quality of the holographic gratings is also of major concern. This study was conducted with two kinds of glass gratings from different makers. One is of $25 \mathrm{~mm} \times$ $25 \mathrm{~mm}$ size and cut into three pieces $(8 \mathrm{~mm} \times 25 \mathrm{~mm}$ each). The other one has $12.5 \mathrm{~mm} \times 25 \mathrm{~mm}$ grating surface. The uniformity of the grating pitch and the depth will influence the diffraction effect and will accordingly alter the dc drift and amplitude of the sinusoidal signals. In addition, improper cutting of the glass gratings creates scratches.

3. The quality of the optics, especially the PBS, is essential to the orthogonality of sine and cosine waveforms.

4. The stability of ambient temperature and the ground vibration are also factors which impact on the system accuracy.

For the medium travel test, it conformed to the ISO230-2 standard of the NC machine tool positioning test. The travel was $5 \mathrm{~mm}$ with $1 \mathrm{~mm}$ steps for bi-directional motion in a selected middle region. The results are shown in figure 16. Although the accuracy could approximately be controlled within $30 \mathrm{~nm}$, the error variation was still large. Again, 
Table 3. Positioning accuracy of fine motions (nm).

\begin{tabular}{lrrrrrr}
\hline $\begin{array}{l}\text { Position } \\
(\mathrm{mm})\end{array}$ & Error 1 & Error 2 & Error 3 & Error 4 & $\overline{\text { Error }}$ & $\sigma_{n-1}$ \\
\hline 0 & 0 & 0 & 0 & 0 & 0 & 0 \\
0.1 & -5 & -13 & -17 & -10 & -11.2 & 5.1 \\
0.2 & -19 & -32 & -27 & -28 & -26.5 & 5.5 \\
0.3 & -27 & -8 & -26 & -33 & -23.5 & 10.8 \\
0.4 & -34 & -33 & -33 & -26 & -31.5 & 3.7 \\
0.5 & -11 & -14 & -20 & -13 & -14.5 & 3.9 \\
0.6 & 0 & -11 & -20 & 21 & -2.5 & 17.7 \\
0.7 & 17 & 2 & -16 & 4 & 1.7 & 13.6 \\
0.8 & 27 & 4 & 3 & 32 & 16.5 & 15.1 \\
0.9 & 14 & 15 & 5 & 23 & 14.3 & 7.4 \\
1 & 7 & 29 & 19 & 4 & 14.8 & 11.5 \\
\hline
\end{tabular}

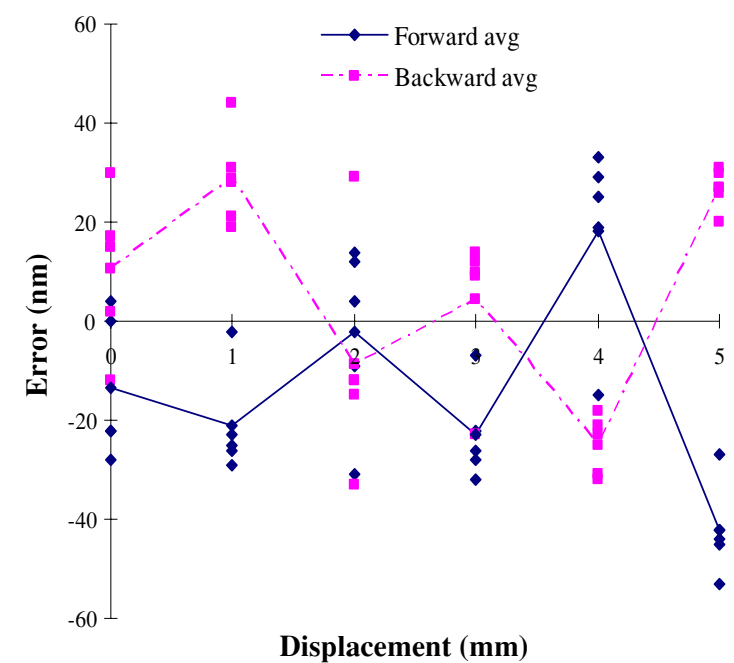

Figure 16. Medium travel positioning accuracy of one axis.

the temperature effect could be the major source, which not only varies the LDGI readings but also those of the laser interferometer.

For the fine motion test of $1 \mathrm{~mm}$ travel with $100 \mu \mathrm{m}$ steps for four runs, the averaged error could possibly fall into the $30 \mathrm{~nm}$ range with only one exception, as shown in table 3 . Since the tested position was specially selected, the error sources of long travels did not exist because there was no missing pitch count of the gratings. However, although the duration of this test was short, the variation of the HP laser interferometer readings could not be eliminated. The temperature variation might dominate the errors.

\subsection{Focus probe test}

The focus error signal (FES) test was carried out by mounting the probe on a linear stage. The stage motion was measured by a laser interferometer. Figure 17 shows the tested results with respect to different materials. A good S-curve occurs when the material has a good reflective surface [14].

\subsection{Spindle motion tests}

A small piece of mirror was mounted onto the table top of the co-planar stage as an object. Initially, the spindle was

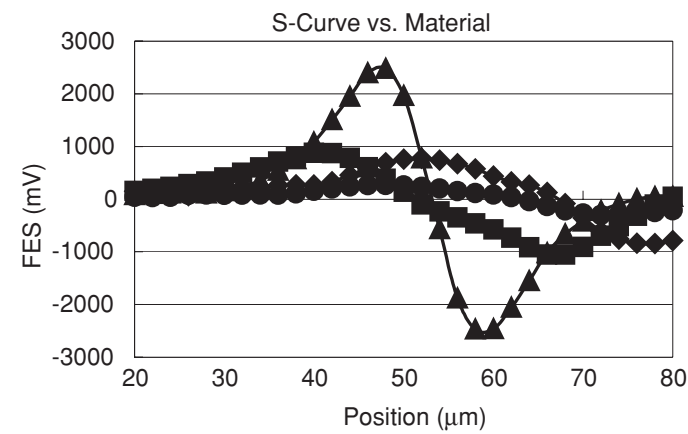

Figure 17. S-curves for various materials.

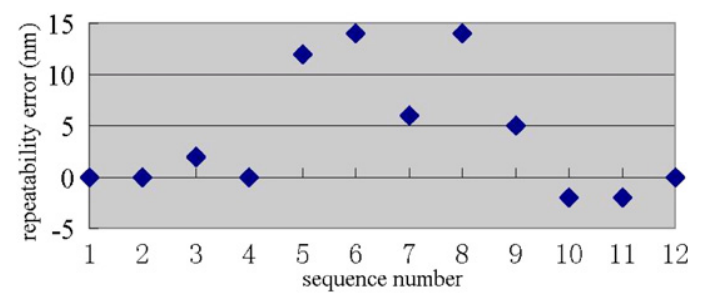

Figure 18. Repeatability test of spindle and probe system.

moved to focus the probe beam right on the mirror surface so that the FES output was zero voltage. The spindle was then moved away a random distance each time and returned until the FES was zero again. The PT-1002A laser interferometer was mounted to detect the spindle motion. The approaching speed for the focus point detection should be very slow in order to avoid any induced dynamic errors. In practice, from a far distance (several millimetres) the approaching speed was about $1 \mathrm{~mm} \mathrm{~s}^{-1}$; within $100 \mu \mathrm{m}$ the speed was reduced to $20 \mu \mathrm{m} \mathrm{s}^{-1}$; within $100 \mathrm{~nm}$ the dc mode of the ultrasonic motor was used with about $10 \mathrm{~nm}$ per step. The readings of the laser interferometer at each focus position showed quite a good repeatability within $15 \mathrm{~nm}$ for 12 runs, as shown in figure 18. As indicated in figure 15, PT-1002A has smaller fluctuation with the time span; the spindle motion is quite successful and reliable.

\section{Concluding remarks}

This paper states the current progress of the development of a micro-CMM. Design considerations and preliminary 
Table 4. Comparison of some small CMMs.

\begin{tabular}{|c|c|c|c|c|}
\hline Institute (item name) & $\begin{array}{l}\text { Measuring range } \\
(X \times Y \times Z)\left(\mathrm{mm}^{3}\right)\end{array}$ & $X-Y($ or $Z)(\mathrm{nm})$ & Overall specs (nm) & Probe \\
\hline $\begin{array}{l}\text { Tokyo University } \\
\text { (nano-CMM) }\end{array}$ & $10 \times 10 \times 10$ & Repeatability: 20 & Accuracy: 50 & $\begin{array}{l}\text { Optical probe } \\
\text { Diameter: } 50 \mu \mathrm{m} \\
\text { Resolution: } 10 \mathrm{~nm} \\
\text { Probing force: } 1 \mathrm{mN}\end{array}$ \\
\hline $\begin{array}{l}\text { SIOS (nano } \\
\text { measuring machine) }\end{array}$ & $25 \times 25 \times 5$ & $\begin{array}{l}\text { Resolution: } 1.24 \\
Z \text {-axis: } 0.1\end{array}$ & Uncertainty: 8-10 & $\begin{array}{l}\text { Focus sensor } \\
\text { Spot diameter: } 0.8 \mu \mathrm{m} \\
\text { Maximum uncertainty: } 2 \mathrm{~nm}\end{array}$ \\
\hline NIST (MMM) & $50 \times 50$ & Resolution: $<1$ & Accuracy: 10 & STM \\
\hline NPL (small CMM) & $50 \times 50 \times 50$ & Resolution: 0.31 & Uncertainty: $50-100$ & $\begin{array}{l}\text { Touch analogue } \\
\text { Diameter: } 0.3 \text { or } 1 \mathrm{~mm} \\
\text { Probing force: } 0.2 \mathrm{mN} \\
\text { Resolution: } 3 \mathrm{~nm}\end{array}$ \\
\hline PTB (special CMM) & $25 \times 40 \times 25$ & Repeatability $<10$ & Uncertainty $<100$ & $\begin{array}{l}\text { Optical fibre } \\
\text { Diameter: } 25 \mu \mathrm{m} \\
\text { Probing force: few } \mu \mathrm{N} \\
\text { Uncertainty: } 80 \mathrm{~nm}\end{array}$ \\
\hline $\begin{array}{l}\text { Eindhoven University } \\
\text { (high-precision } \\
\text { 3D-CMM) }\end{array}$ & $100 \times 100 \times 100$ & Uncertainty $<100$ & Uncertainty $<100$ & $\begin{array}{l}\text { Touch analogue } \\
\text { Diameter: } 0.3 \mathrm{~mm} \\
\text { Probing force }<1 \mathrm{mN} \\
\text { Resolution: } 1 \mathrm{~nm} \\
\text { Uncertainty: } 25 \mathrm{~nm}\end{array}$ \\
\hline $\begin{array}{l}\text { NTU/HFUT } \\
\text { (micro-CMM) }\end{array}$ & $20 \times 20 \times 10$ & $\begin{array}{l}\text { Repeatability } \\
\text { in } X-Y: 30 \\
\text { in } Z: 15\end{array}$ & Not completed & $\begin{array}{l}\text { Focusing } \\
\text { Spot diameter: } 0.8 \mu \mathrm{m} \\
\text { Resolution: } 1 \mathrm{~nm}\end{array}$ \\
\hline
\end{tabular}

results are described. With particular consideration of the structural accuracy, new ideas for an arch bridge and the co-planar stage are proposed. Equipped with the SP4 ultrasonic actuator and the LDGI feedback system the motions in the $X$ and $Y$ directions can achieve $1 \mathrm{~nm}$ resolution, the same as the $Z$-spindle motion mounted with the focus probe when driven by SP-4 and detected by the PT-1002A laser interferometer. Experimental tests have shown that the $Z$-axis and the fine motion of the co-planar stage can meet the required accuracy of below $30 \mathrm{~nm}$. The medium and long motions of the co-planar stage are still slightly over the requirement. Some possible error sources have been discussed. The whole software system of this prototype micro-CMM is developed in the LABVIEW environment. In the current work, the system integration and tuning process has not been completed. All tested data presented in this paper only fit to our current environment condition $\left(20 \pm 1{ }^{\circ} \mathrm{C}\right)$. Further experiments in a stricter condition of better than $20 \pm 0.1^{\circ} \mathrm{C}$ will be carried out after the construction is completed. Comparisons with other existing systems are listed in table 4 . It has to be noted here that data collected in this table are obtained from the references of this paper. Updated data are not available. The probing system data are mostly quoted from [21]. Continued work will focus on the improvement of the straightness of the guide way by harder workmanship, the proper selection of the holographic gratings as well as the optics for better output signals and the construction of a mini temperature and humidity controlled chamber to protect the system environment. Moreover, the volumetric errors of the CMM have to be calibrated and compensated in order to achieve higher accuracy. In addition, the miniature contact probe will be studied.

\section{Acknowledgments}

The present authors gratefully acknowledge the support provided to this project by the National Natural Science Council of China under contract nos. 50275048 and 50420120134, and the National Science Council of Taiwan under contract no. 942212E002001.

\section{References}

[1] McKeown P 1998 Nanotechnology-special article Proc. Nano-metrology in Precision Engineering (Hong Kong, 24-25 November) pp 5-55

[2] Takamasu K, Guo B W, Furutani R and Ozano S 1998 Basic concept of feature-based metrology Proc. 6th ISMQC Symp. (Vienna)

[3] Ni J 2004 Future direction of micro/meso-scale manufacturing Invited speech, Proc. 6th ICFDM'2004 (Xi' an, China, 21-23 June)

[4] Peggs G N, Lewis A and Leach R K 2003 Measuring in three dimensions at the mesoscopic level Proc. ASPE Winter Topical Meeting-Machines and Processes for Micro-scale and Meso-scale Fabrication, Metrology and Assembly (FL, USA, January 2003) pp 53-7

[5] Hausottee T et al 2004 Traceable nanometrology with a nanopositioning and nanomeasuring machine J. Chin. Soc. Mech. Eng. 25 399-404

[6] Fan K C, Chu C L, Chang S H and Chung T T 2001 Development of a micro-CMM for nanometrology Proc. KSPE Spring Conf., Keynote paper pp 1-6

[7] Liang S 2004 Machining and metrology at micro/nano scale Keynote speech Proc. 1st ICPT (Hamamatsu, Japan, 9-11 June 2004)

[8] Enami K, Hiraki M and Takamasu K 2000 Nano probe using optical sensing 16th IMEKO World Congress, IMEKO 2000 (Vienna, Austria, September 2000) 
[9] Haitjema H, Pril W O and Schellekens P 2001 Development of a silicon-based nanoprobe system for 3-D measurements Ann. CIRP $50365-8$

[10] Schwenke H, Härtig F, Wendit K and Wäldele F 2001

Future challenges in co-ordinate metrology: addressing metrological problems for very small and very large parts IDW Conf. (Knoxville, TN) pp 1-12

[11] Pohl D W 1987 Dynamic piezoelectric translation devices Rev. Sci. Instrum. 58 54-7

[12] Fan K C 2002 A high precision diffraction interferometry stylus probing system Keynote paper Proc. ICMT (Kitakyushu, Japan)

[13] Fan K C, Chu C L and Mou J I 2001 Development of a low-cost autofocusing probe for profile measurement Meas. Sci. Technol. 12 2137-46

[14] Fan K C, Lin C Y and Shyu L H 2000 Development of a low-cost focusing probe for profile measurement Meas. Sci. Technol. 11 1-7
[15] Fan K C, Wang W L and Chen F 2004 Innovative design of a new CMM bridge Proc. ISPMM 2004 (Beijing, China, August 2004) paper no 50

[16] Heydemann P L M 1981 Determination and correction of quadrature correction of fringe measurement errors in interferometers Appl. Opt. 203382

[17] Birch K P 1990 Optical fringe subdivision with nanometric accuracy Precis. Eng. 12 195-8

[18] Chen B Y and Li D C 2002 Progress in studies on long-range and ultrahigh-accuracy nanometer measurements Proc. 2nd ISIST (Jinan, China) vol 2 pp 84-9

[19] Wen P and Hsu D H 1990 Direct subdivision of moiré fringe with CCD Proc. SPIE $1230165-6$

[20] Burton G L and Burton P J 1996 X-Y-Theta positioning mechanism US Patent No. 5523942

[21] Weckenmann A, Peggs G and Hoffmann J 2005 Probing systems for dimensional micro and nano metrology Proc. ISMTII 2005 (Huddersfield, UK, September 2005) pp 199-206 